ACAMH for Child and Adolescent

Mental Health

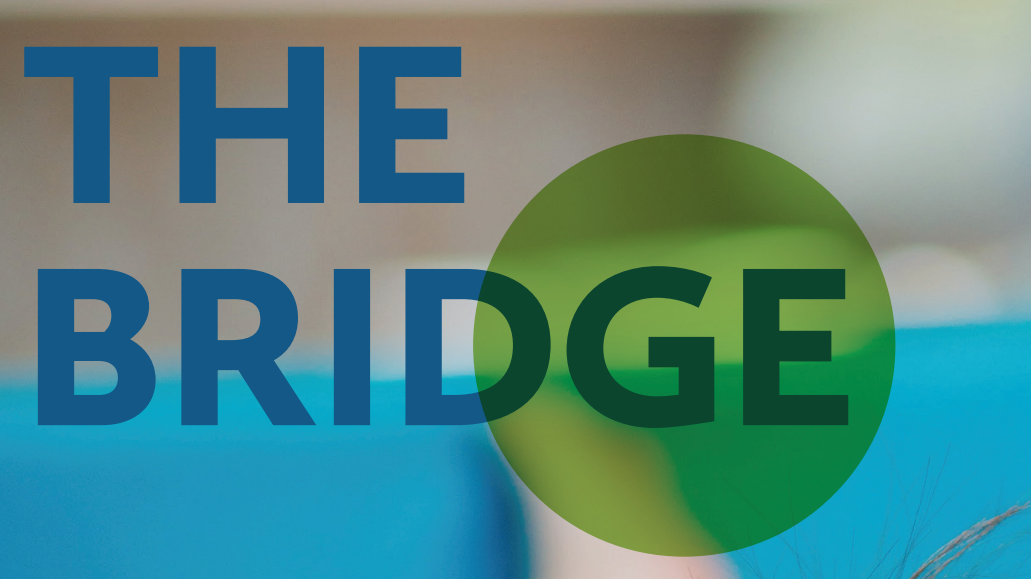

Informatics

Issue

FEB 2018

Are digital health interventions for young people meeting expectations?

Review of CAMH \& JCPP Papers

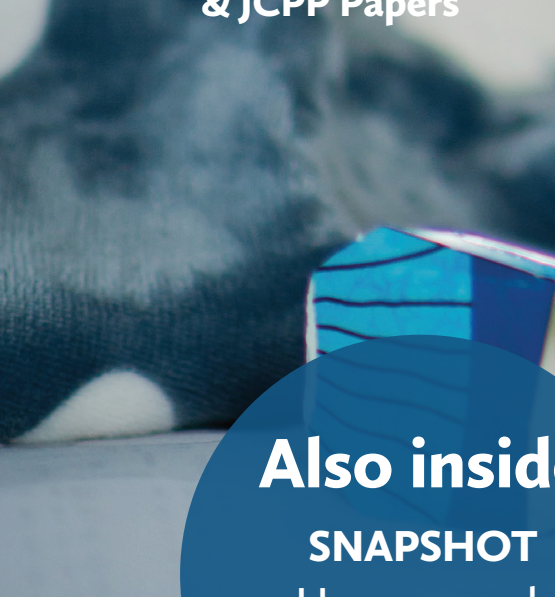

How research on cyberbullying has developed 


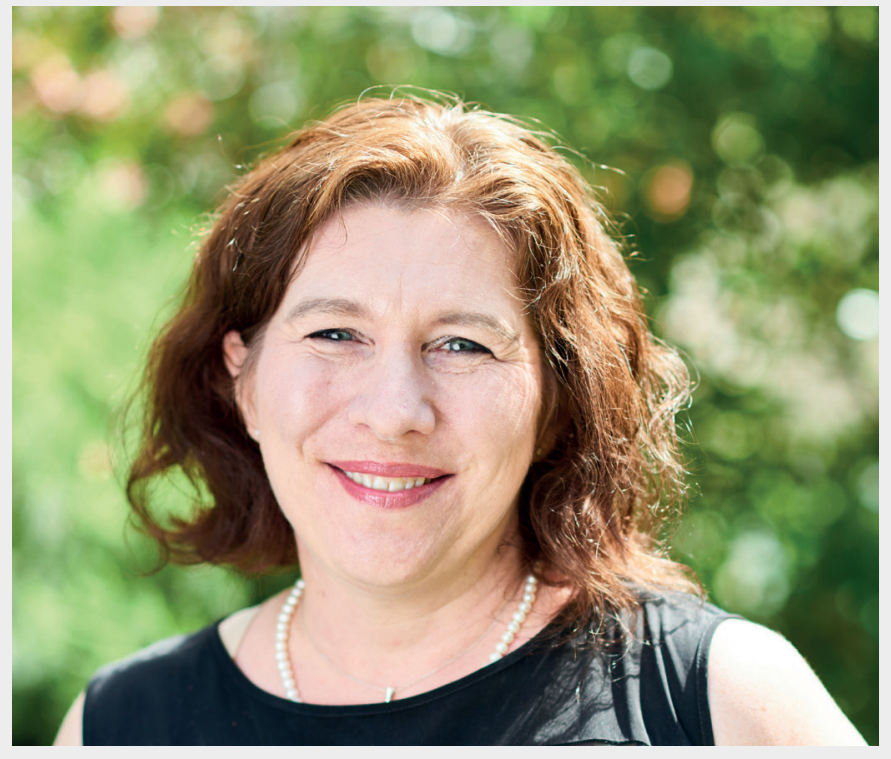

\section{Foreword from the Editor}

Welcome to this informatics themed edition of the Bridge. It is rare today to see a young person without a smartphone in their hand or pocket. Young people have increasing access to technology which creates many opportunities and challenges. Apps and websites can deliver psychoeducation and even therapy, some examples and their evaluation are outlined in this edition. Social media platforms can connect people but can also be a medium for bullying. The Autcraft platform provides a novel way for children with ASC to make friends, but also highlights how important it is that parents and carers are supported in understanding children's online activity, so that, safe helpful, online spaces can be created and maintained. The informatics field is advancing rapidly so we will revisit it regularly in The Bridge.

\section{Dr Juliette Kennedy}

Editor of The Bridge

\section{iPad App complements ASD therapy}

\author{
Summarised by Dr. Jessica K Edwards
}

Children with autism spectrum condition (ASC) may benefit from combined technology-based and traditional interventions, according to new research. Andrew Whitehouse and colleagues assessed the efficacy of TOBY (Therapy Outcomes By You) in a randomised controlled trial (ACTRN12614000738628). This is an iPad application to help children and carers structure home-based therapy. The trial included 80 children within 12 months of an ASC diagnosis, who were assigned their usual therapy or usual therapy plus TOBY. Significant improvements were identified at 6-months follow up for secondary outcomes (visual reception and fine motor skills, and single-word comprehension) but not for primary outcomes (Autism Treatment Evaluation Checklist). Although those assigned to use TOBY did not effectively sustain its use after the first 3 months of the trial, $70 \%$ of carers reported that TOBY was "a helpful therapy planning tool". The overall exposure to therapy per child increased, as a result of using TOBY, with minor cost implications.

Whitehouse, A. J. O., Granich, J., Alvares, G., Busacca, M., Cooper, M. N., Dass, A., Duong, T., Harper, R., Marshall, W., Richdale, A., Rodwell, T., Trembath, D., Vellanki, P., Moore, D. W. \& Anderson, A. (2017), A randomised controlled trial of an iPad-based application to complement early behavioural intervention in Autism Spectrum Disorder. J Child Psychol Psychiatr, 58:10421052. doi:10.1111/jcpp.12752 


\section{Minecraft \\ for young \\ people with Autism}

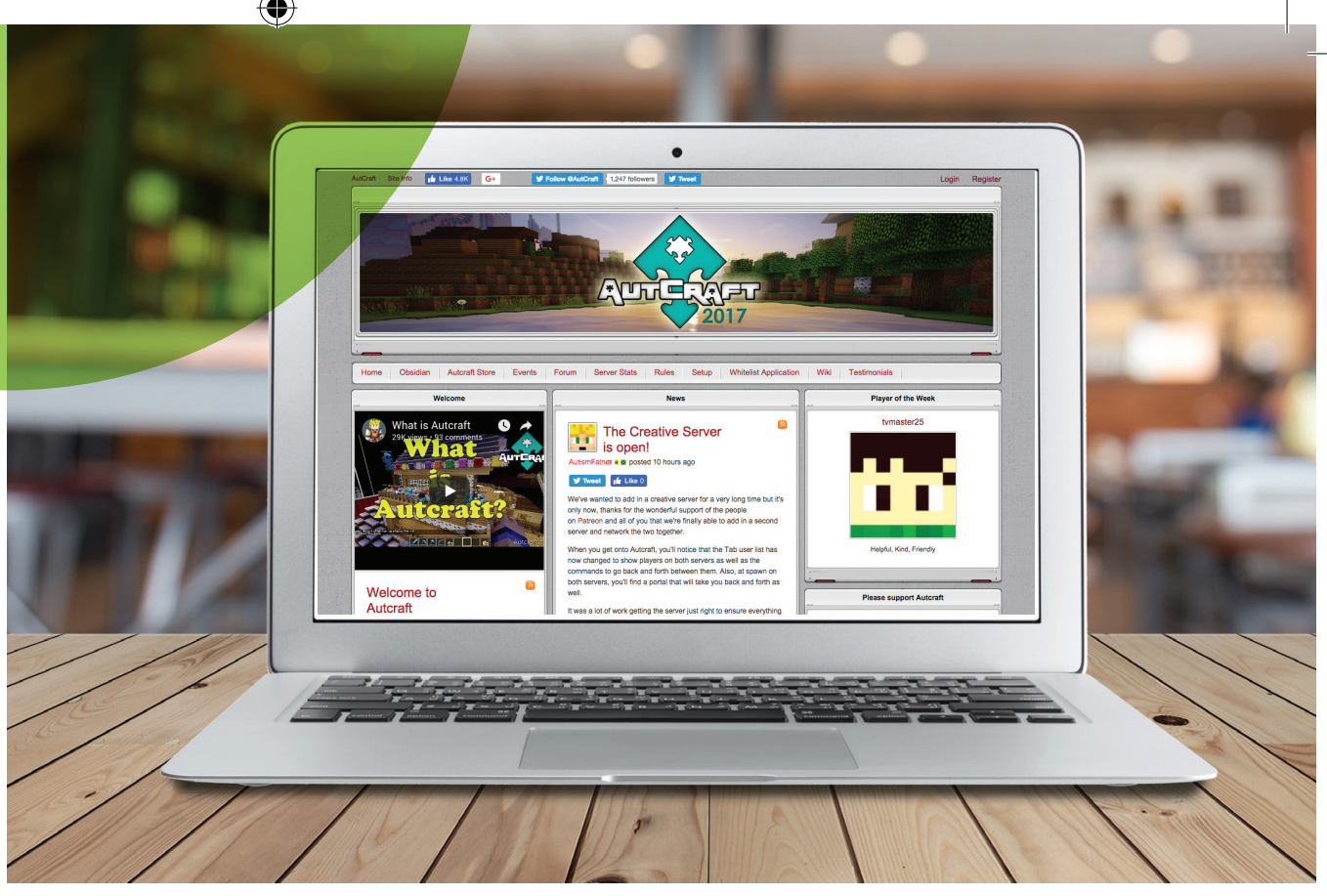

By Ben Upton

\section{The Bridge spoke to Stuart Duncan, who has developed - Autcraft - Minecraft for young people with Autism}

In June 2013, Stuart Duncan, a

Canadian web developer and blogger, posted on his Facebook page the idea of having a dedicated place on the internet for children and young people with autism to play.

Stuart was diagnosed with Asperger's syndrome when he was 36 and has two sons. His eldest, Cameron, also has autism.

Stuart and his sons have enjoyed playing the computer game Minecraft together for many years, but for other Minecraft players with autism, trying to play with others can often result in insults and threats from strangers online.

"Kids with autism would go onto public servers to play Minecraft and they would just be bullied," said Stuart.

The wildly popular sandbox-style game is often described as digital lego, and has proved a hit with adults and children worldwide, including those with autism.

"I've often heard it said that people with autism gravitate towards it because all the rules of the world are set and predictable," said Stuart.

He thinks Minecraft also appeals to children with autism because they often become discouraged by the prescriptive nature of other games. "There are no guidelines - you're free to do whatever you want" he said.
Stuart set up the server, known as Autcraft, with a 'whitelist' which limits access to those with autism diagnoses and their families. "I got 750 emails in the first 2 days," he said.

Four years on, the whitelist now includes over 9000 members, with around 1200 signing in to play each month, and around 30-40 players playing together at any one time.

Since its creation, this world has been gently policed by a team of volunteer moderators, recruited from Stuart's friends.

Swelling membership has called for new recruits, with all volunteers coming from the global community of Autcraft players. "There're kids with autism, siblings, adults with autism, adults without autism, l've even had grandparents get involved," he said.

3 days ago we had 3 sets of kids playing with their parents at the same time" said Stuart "a few parents had never played before and have since become admins."

These admins can mute people, temporarily jail them in an inescapable room in the game or even ban players from the server outright for serious violations of the server rules. These are fairly mild, and mainly focussed on creating a safe, friendly environment for younger players.
Stuart actively recruits parents to join the server and play with their children, both directly and by posting invitations on social media. "It's a terrific bonding experience," he said, explaining that a child's previously incomprehensible Minecraft chat can become a shared source of fun, if parents give it a go.

Stuart said one of the big criticisms of the server has been that autistic children should be interacting face to face, not online. His response is that something is better than nothing "at least they're socialising and making friends," he said.

In addition, the Autcraft players have had a few physical meet ups in the US, where events draw up to 50 players and their parents, who go on outings, and of course, play Minecraft.

"There are these two girls in Australia - they live 14 hours' drive away from each other" Stuart said "one had a wicked temper, the other was more shy."

" 2 years later one of them took the drive with her parents, just to meet the other face to face. They came back and told me they'd never had a friend before," he said.

Stuart believes the Autcraft server has helped a lot of players to socialise more, and he has received messages from parents saying exactly that.

To read more, please visit www.autcraft.com 


\section{Digital health interventions}

for the young: meeting

\section{expectations?}

By Dr. Jessica K Edwards

The number of digital health interventions for mental health disorders is increasing, but research from Chris Hollis and colleagues suggests that the clinical benefits and costeffectiveness are unclear. Consistent methods of reporting and evaluation are required to extract definitive conclusions from clinical trials.

The enthusiasm of children and young people (CYP) towards digital technology has spurred the rapid development of digital health interventions (DHIs) for this age group. For mental health disorders, DHIs have the potential to reach CYP at a relatively low cost, through a medium that is presumed accessible and familiar, whilst helping overcome access barriers to treatment. However, whether the proposed benefits of DHIs are truly realised is unclear. Now, a comprehensive review of the literature has found that DHIs have some clinical benefit for those with depression and anxiety but evidence supporting their use in attention deficit hyperactivity disorder (ADHD), autism spectrum disorders (ASD), psychosis and eating disorders is lacking.

In a review paper published in the Journal of Child Psychology and Psychiatry, Chris Hollis and his colleagues at the University of Nottingham, University College London and the Warneford Hospital, Oxford assessed the evidence for clinical benefit and cost-effectiveness of DHIs used by CYP with mental health problems, by synthesising data from previous reviews (meta-review) and providing an updated systematic review of randomised controlled trials (RCTs). Their meta-review included 21 articles, containing data from 190 trials of $\sim 147$ different DHls. Their systematic review included 30 RCTs for DHls aimed at improving outcomes in ADHD, ASD, psychosis, anxiety, depression, anxiety and depression, eating disorders and PTSD and depression, which between them randomised $>5,000$ participants.

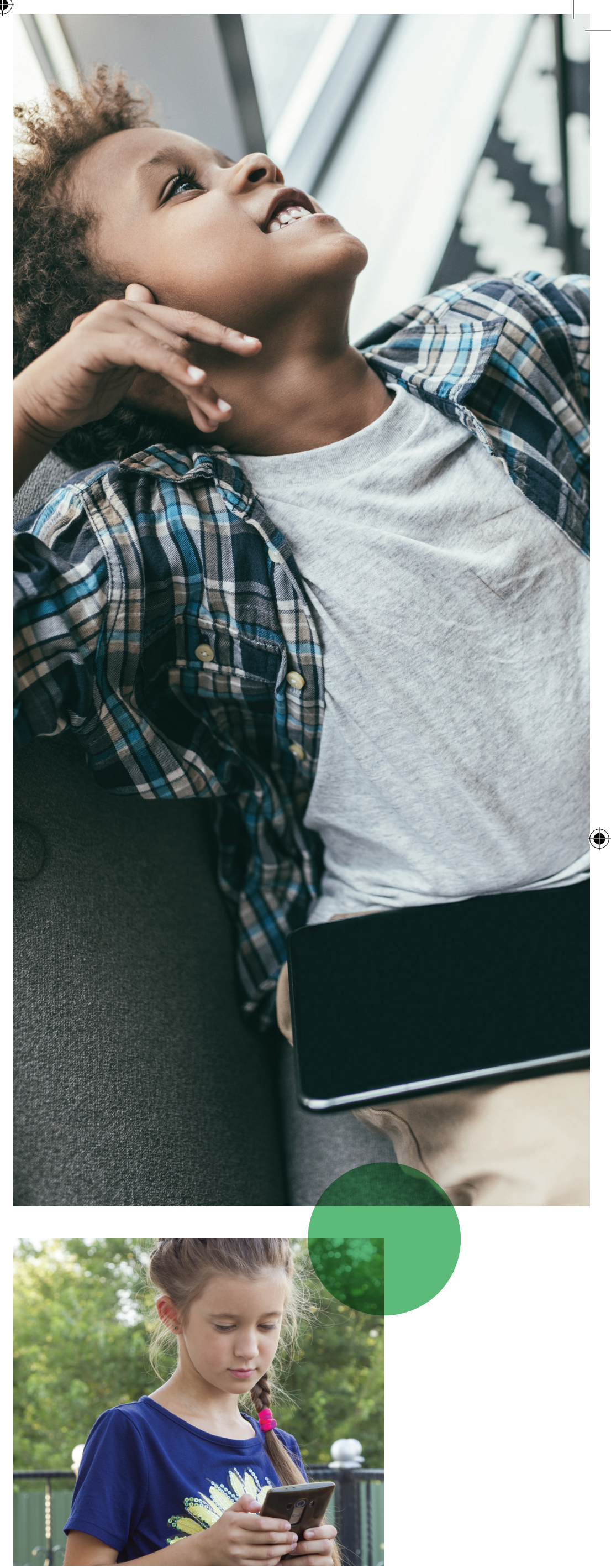




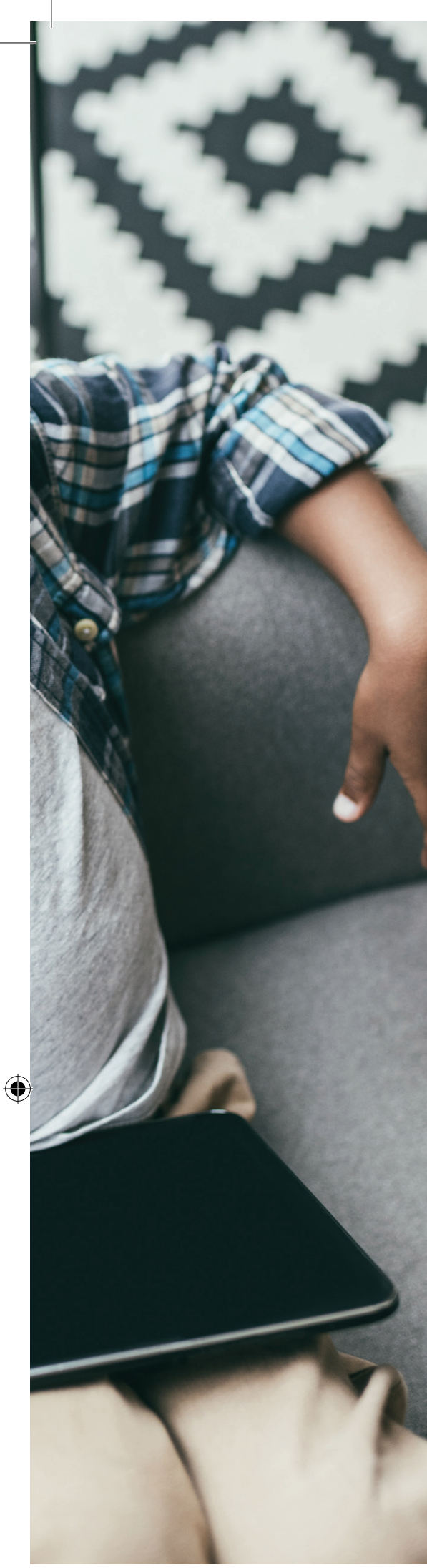

Ref: Hollis, C., Falconer, C. J., Martin,J. L., Whittington, C., Stockton, S., Glazebrook, C., Davies, E. B. (2017), Digital health interventions for children and young people with mental health problems - a systematic and meta-review. J Child Psychol Psychiatr, 58: 474-503. doi: 10.1111/jcpp.12663
According to their analyses, depression and anxiety are the most common clinical targets for DHls used by CYP, with the majority of these DHIs using an online, "sessional" computerised cognitive behavioural therapy (cCBT) format. In fact, cCBT achieved the strongest evidence of clinical effectiveness for depression and anxiety in adolescents and young adults. However, Hollis et al note that cCBT is limited by its "sit-down approach to treatment" and that it fails to exploit the full capabilities of modern digital technologies. "MobileType", however, is one $\mathrm{DH}$ that does exploit the "ubiquitous nature of mobile technologies", using a momentary sampling approach to assess mood, stress, current activity, alcohol and cannabis use. The researchers propose that although not therapeutic, this type of utility may help personalise DHIs and support adherence. Conversely, Hollis et al found that selfguided interventions, such as "MoodGYM", tend to achieve poor uptake and adherence. Finally, the researchers found no studies of DHIs for CYP with depression and anxiety that included data on cost-effectiveness.

The effectiveness of DHls for ADHD in CYP was not assessed in any of the included studies. Three main interventions were identified: electroencephalogram (EEG)-based neurofeedback training (NFT), executive functioning training (EFT) and working memory training (WMT). EEG-NFT aims to decrease theta brain-wave activity and increase beta wave activity, while WMT and EFT aim to improve deficits in cognitive function and attention. Inconsistent results were reported for these interventions, with no overall differences found between the $\mathrm{DHI}$ and active placebo intervention group. From their updated systematic review, Hollis et al confirm that these DHls cannot yet be recommended for ADHD treatment and conclude that "given that face-to-face nonpharmacological interventions for ADHD have not demonstrated efficacy, it is perhaps not surprising that digital versions would also not be effective".

The DHIs for ASD typically use a computer game-based format designed for preadolescent children and aim to improve on core deficits in social understanding, empathy and emotional recognition. Unfortunately, trials have not yet demonstrated a beneficial transfer of skills out of the "game" context to affect core ASD symptoms. Hollis et al propose that DHIs that focus on associated symptoms and behaviours in ASD, such as anxiety, may be more amenable to intervention than those that focus on the core deficits.

According to Hollis et al, various issues should now be addressed to maximise the potential, adherence and clinical benefit of DHIs in the mental-health setting. DHIs have the potential to deliver effective interventions on a wide scale at minimal cost relative to traditional therapies; it is therefore noted by the researchers that lack of data on their cost-effectiveness is "surprising given the promise that DHIs can increase health service efficiency". Estimations of cost seem to be complex, requiring considerations of human support, the location where the $\mathrm{DHI}$ is delivered and the ownership of the DHI (i.e. whether DHIs are commercialised or free-to-use). Hollis et al emphasise that DHI sustainability and cost-effectiveness should be considered at the outset of DHI development and that "the development phase for a DHI should include consideration of the long-term costs of maintenance and updating, how these costs could be met and who will take responsibility for them".

As well as cost implications, human support also compounds a key benefit of DHls -automated delivery. Hollis et al found that the level of human support is poorly specified in many of the studies analysed. There is wide variation as to who provides the support, and the amount, its purpose and uniqueness. Further studies that address the benefits of human support on adherence and outcomes of DHIs in CYP are needed. The researchers suggest that human support may not necessarily be delivered by an actual person, but that "virtual human agents" could be a possibility in next generation DHIs.

To read more, please visit http://bit.ly/2hMıVeq 


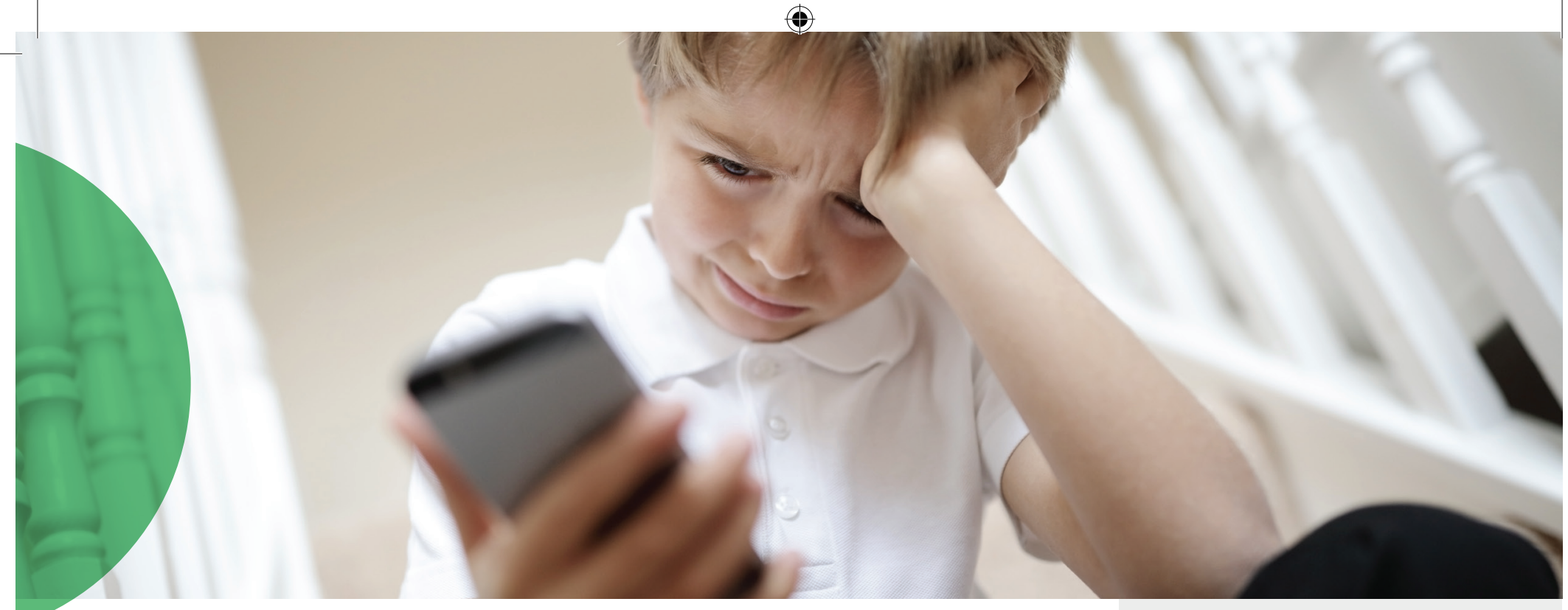

\section{SNAPSHOT}

\section{How research on cyberbullying has developed}

Prof. Peter K. Smith

Goldsmiths, University of London, England

The topic of cyberbullying is often in the media, because of the distress and harm it can cause. There have been cases where it appears to have contributed significantly to tragic outcomes such as suicide (Livingstone \& Smith, 2014). According to Sheri Bauman, the earliest use of the term dates back to a New York Times article in 1995. But it took a few years to catch on. Probably the earliest website was created by Bill Belsey in 2003, at www.cyberbullying.ca. That website is still there (and claims to have made the first definition of cyberbullying) but now it is joined by many others.

Research on the topic started in the early years of this century, first in North America. For example Michele Ybarra reported on internet harassment. An analysis by Fethi Berkkun and myself (Smith \& Berkkun, 2017) used the Web of Science database to extract details of articles on cyberbullying. This shows how the number of articles has built up from a very slow start during the early years of this century to a rapid increase. The year 2015 saw 131 articles, or about 2.5 new articles every week, and this shows no signs of abating. We also found that for the first decade, the majority of research came from North America, but in recent years the European countries have produced the most research, perhaps stimulated by the possibility of EU funding.

In Europe, a relatively early study by myself and colleagues (Smith et al., 2008) described the nature and prevalence of cyberbullying at that time. Until then, most cyberbullying was by emails, or by text messages or prank calls on mobile phones. Smart phones were yet to come. But the next few years saw modes of cyberbullying change dramatically. Many young people started to use smart phones to access the internet. They also started to use services such as instant messaging and a variety of social networking sites such as Facebook, Instagram, Snapchat and Twitter became very popular. Apart from internet gaming, social networking appears to be somewhat more prevalent in girls than boys, and cyberbullying experiences too seem at least relatively more common in girls, compared to what is now called 'traditional', or offline bullying.

Obviously, cyberbullying is an international issue. Although coping and resilience vary, it clearly can have a very negative effect. Debate is ongoing about definition (should we use the term cyber-aggression?), the extent of overlap with traditional bullying, and how best to help schools and young people (where the problem seems most acute). Campbell and Bauman (2018) provide a compendium of international contributions, on what can be done.

For the full length article, please visit http://bit.ly/2hMıVeq

Key

\section{Practitioner}

\section{Messages}

- Being a victim of cyber attacks or cyberbullying can cause great distress and harm - as much or more than traditional bullying

- Children with preexisting vulnerabilities will be more at risk of harm; those with better digital skills and more social support are likely to cope better

- Parents have a role in being interested in and monitoring young people's internet use, without being too intrusive

- Schools should mention cyberbullying in their anti-bullying policy, and cover internet safety issues through the curriculum

- Many websites provide useful advice - see for example the AntiBullying Alliance website at https://www.antibullyingalliance.org. uk/tools-information/ all-about-bullying/ cyberbullying-o 


\section{Designing services with patients calls for curiosity and empathy}

By Ben Upton

Dr Victoria Betton is a social worker by training and has spent many years working in the NHS and with her local authority, before working in public involvement in the NHS using digital technologies. She currently leads mHabitat, a digital innovation team that is part of Leeds and York Partnership NHS Foundation Trust.

"I took my knowledge from the NHS about involving patients and combined that with design tools from commercial software development," she said,

"What we do is co-design. We design things collaboratively with the people that are going to end up using them" said Victoria "we believe you end up with better products and services that people want and like," she said.

"We've usually got an idea or a problem that needs solving, then we involve end users, young people, families and practitioners in designing solutions to that problem together."

She said that in the NHS, people often only involve service users such as young people at the end of the process, when the product or service is already developed.

"The difference in co-design is you bring people together, often in a workshop environment, and use creative processes to come up with design ideas against a brief," she said.
When working with young people, they often make use of drawing and tactile toys like Play Doh to explore ideas.

"If there's a digital focus then we'd actually be getting paper and scissors and designing paper versions of an app," she said.

For those interested in co-design, Victoria recommends the 'DIY Toolkit' a service design guide developed by Nesta UK, a charity which supports innovation. "They've got free tools you can just download and use in your project," she said.

Victoria said the team often begins by asking the workshop if they're actually asking the right question in the first place, she gave the example of a project where a service that used mainly phone calls and letters wanted to improve young people's access and communication with CAMHS.

The mHabitat team assumed that because young people might have to travel quite far to get to a consultation, they'd prefer a video consultation instead. But, after speaking to them, they found out that a lack of privacy at home meant many would rather receive the cost of the bus fare electronically allowing them to come and see a consultant in person.

To read more, please visit http://wearemhabitat.com/

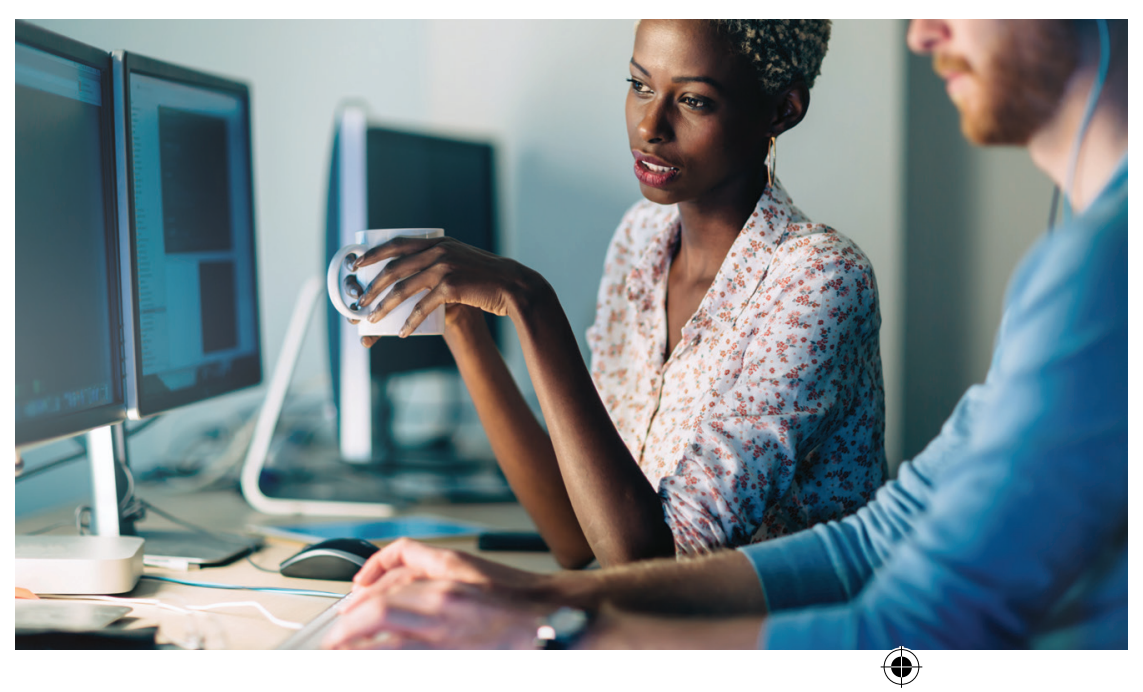

mHealth ineffective for

\section{depression prevention}

Summarised by Dr. Jessica K Edwards

A universal cognitive behavioural therapy (CBT)-based mobile messaging programme (MEMO CBT) designed to prevent teenage onset depression provides no clinical benefit, according to results of a double-blind, randomised, placebo-controlled trial (ACTRN12609000405213). A total of 855 students (recruited from 15 schools across Auckland, New Zealand) received text and multimedia mobile-phone messages for 9 weeks based on key principals of CBT (MEMO CBT) or unrelated content (MEMO control). No significant differences in primary (CDRS-R score) or secondary (including RADS-2, MFQ or PQ-LES-Q scores) outcomes were identified between the two groups at 12 months follow-up. The researchers suggest that the healthiness of the participants and the low level of CBT exposure may have contributed to these negative findings. Further work is required to demonstrate the value of universal prevention interventions via mHealth modalities for depression.

Whittaker, R., Stasiak, K., McDowell, H., Doherty, l., Shepherd, M., Chua, S., Dorey, E., Parag, V., Ameratunga, S., Rodgers, A. \& Merry, S. (2017), MEMO: an mHealth intervention to prevent the onset of depression in adolescents: a double-blind, randomised, placebo-controlled trial. J Child Psychol Psychiatr, 58:1014-1022. doi:10.1111/jcpp.12753 
The Association

for Child and Adolescent

Mental Health

\section{Emanuel Miller Memorial Lecture and National Conference 2018}

\section{Focusing on Adolescent Mental Health}

Friday 16 March 2018 / The Royal College of Physicians, London

The eagerly anticipated Emanuel Miller Memorial Lecture and National Conference returns in 2018 with a truly outstanding line-up of world renowned practitioners, clinicians and researchers in the field of adolescent mental health.

We are honoured to have Professor Sir Robin Murray deliver the Emanuel Miller Memorial Lecture on the topic of adolescent drug use and its link to psychosis.

The Keynote Speech is presented by Professor lan Goodyer, University of Cambridge, who will be speaking on the topic of neuroscientific approaches to the emergence of major depressions in adolescence.

\section{TO BOOK: 放 www.acamh.org}

(6) +44 (0)20 74037458

$\$$ events@acamh.org

\section{Research Highlights}

\section{Changing perceptions on technological therapy}

Summarised by Dr. Jessica K Edwards

Educating parents in computer-based therapies for youths with mental health disorders may improve uptake of this therapeutic modality by affected families. Parents who viewed a short presentation on computer-based therapies, reported an improved understanding of the rationale, benefits and helpfulness of this technique, compared to those who viewed an unrelated, control presentation. Specifically, those with low confidence in using technology, demonstrated high gains in knowledge and perceived helpfulness, and parents considered "neurotic or less open" perceived fewer problems with this therapy after viewing the presentation. It remains to be determined whether changes in perception to computer-based therapy, made immediately following an educational presentation, are sustained and boost uptake in children.

Sweeney, G. M., Donovan, C. L., March, S. \& Laurenson, S. D. (2017), Can we improve parent attitudes and intentions to access computer-based therapies for their children and adolescents? Child Adolesc Ment Health, 22:155-162. doi:10.1111/ camh.12180

\section{Parents with BD receive online support}

Summarised by Dr. Jessica K Edwards

The value of a unique interactive, web-based resource that provides psychoeducational and parenting information for patients with bipolar disorder (BD) and young children has been supported by promising results of a randomised, controlled pilot trial (ISRCTN75279027). According to the study, which randomised 97 eligible adults from UK NHS trusts, Integrated Bipolar Parenting Intervention (IBPI) is a feasible strategy to help reduce parenting stress, improve sense of competence, and reduce risk of associated child behavioural problems, when used in combination with the patient's usual treatment. Although IBPI achieved a high retention rate ( $90 \%$ ) to the 48 -week follow-up, only $53 \%$ of participants accessed the parenting (Triple P) modules during the trial. Future trials that explore the impact of parental mood, the longevity of the benefits of IBPI on risk of mental health problems in children, and clinical and costeffectiveness are planned.

Jones, S. H., Jovanoska, J., Calam, R., Wainwright, L. D., Vincent, H., Asar, O., Diggle, P. J., Parker, R., Long, R., Sanders, M. \& Lobban, F. (2017), Web-based integrated bipolar parenting intervention for parents with bipolar disorder: a randomised controlled pilot trial. J Child Psychol Psychiatr, 58:1033-1041. doi:10.1111/jcpp.12745 